\title{
Long Non Coding RNA-UCA1 Contributes to Cardiomyocyte Apoptosis by Suppression of p27 Expression
}

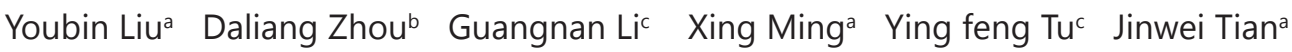 \\ Huimin $\mathrm{Lu}^{\mathrm{d}}$ Bo Yu${ }^{\text {a }}$
}

aKey Laboratories of Education Ministry for Myocardial Ischaemia Mechanism and Treatment (Harbin Medical University), Ministry of Education and Department of Cardiology, The Second Affiliated Hospital, Harbin Medical University, Harbin, bDepartment ofCardiology, The First Hospital of Harbin City, Harbin, 'Department of Cardiology, The Fourth Affiliated Hospital Harbin Medical University, Harbin, dDepartment of Nutrition and Food Hygiene, Public Health College, Harbin Medical University, Harbin, PR China

\section{Key Words}

Long non coding RNA • UCA1 • Cardiomyocyte apoptosis • p27

\begin{abstract}
Background/Aims: Urothelial carcinoma-associated 1 (UCA1) is a recently identified long non coding RNA (IncRNA). However, few studies have explored its role in cardiomyocytes after focal cardiac ischemia reperfusion injury (CIR). Methods: Rat CIR models were established using ligation of the Lower Anterior Descending artery (LAD). Cell apoptosis and reactive oxygen species (ROS) production in cardiac tissues were explored using immunohistochemistry and DHE staining. IncRNA expression patterns were detected using microarray and validated by qPCR. Cell viability and apoptosis were examined using MTT assay and flow cytometry. Results: CIR significantly induced cell apoptosis and ROS production in the rat model. The results of microarray demonstrated the reduced expression of UCA1, which was validated by qPCR. Follow-up experiments showed that UCA1 was involved in $\mathrm{H}_{2} \mathrm{O}_{2}$-induced cell apoptosis. We further showed that UCA1 negatively correlated with the expression of p27. Moreover, overexpression of p27 could induce primary cardiomyocyte apoptosis. Conclusions: Reduction of UCA1 levels plays a pro-apoptotic role in primary cardiomyocytes partially through stimulation of p27 protein expression. These results are in agreement with the observed levels of UCA1, p27 and apoptosis after cardiac I/R injury, suggesting that UCA1 might have an important role during I/R injury.
\end{abstract}

Y. Liu, D. Zhou and G. Li contributed equally to the work.

Bo Yu

and Huimin Lu

KARGER 125
The Key Laboratories of Education Ministry for Myocardial Ischemia Mechanism and Treatment, Department of Cardiology, The Second Affiliated Hospital, No. 246 Xuefu Street, Nangang District, Harbin City, Heilongjiang Province, 150001 (PR China)

E-Mail Yubodr@163.com and E-Mail lhm_519@sina.com 


\section{Introduction}

Ischemic heart disease, a leading cause of death worldwide, is the most common consequence of coronary artery disease [1]. In the disease pathology, myocardial infarction and angina pectoris are key factors, accompanied by changes in gene expression [2]. At present, long noncoding RNAs (lncRNAs) are theorized to play key roles in the process of ischemic heart disease (IHD) [3]. IncRNAs are typically defined as noncoding RNA molecules ranging in length from $200 \mathrm{nt}$ to $\sim 100 \mathrm{~kb}$ and lacking a protein-coding ability [4]. Through a variety of mechanisms, IncRNAs are reported to play key roles in the pathophysiology of some diseases such as Alzheimer's disease and cancer [5]. They mainly function as scaffolds for multiple proteins, thereby forming complexes that regulate protein expression [6].

Cardiomyocyte death is an important contributor to cardiac syndromes such as heart failure (HF), myocardial infarction (MI) and stroke [7]. Although reperfusion of an occluded human coronary is effective for reducing overall mortality, it is now recognized that restoration of the blood flow through the previously ischemic myocardium can yield additional reperfusion injury, including cardiomyocyte dysfunction and cell death. The cellular mechanisms underlying ischemia/reperfusion (I/R)-induced injury are complex and involve a multitude of signaling pathways and molecular players. Recent studies have demonstrated that apoptosis plays a key role in ischemia/reperfusion (I/R)-induced injury [8]. These studies show that apoptosis plays an important role in the pathological process in cardiac diseases. However, the molecular components involved in regulating programmed apoptosis in heart tissue remain largely unidentified.

As a tumor suppresser, p27 significantly inhibits the expression of cyclin-dependent kinase [9]. In the ischemic heart, p27 is frequently upregulated, and reduced p27 expression correlates with reduced cell proliferation [10]. The mechanism that controls p27 expression is complex, occurring at the transcriptional, translational and post-translational levels [11, 12]. p27 is reported to contribute to the resistance to cell death primarily through cell cycle arrest or through other less defined mechanisms [10]. In addition, p27 leads to the activation of cleaved-caspase 3 (c-caspase3), thereby triggering cell apoptosis.

Urothelial carcinoma-associated 1 (UCA1) is a IncRNA originally identified in bladder transitional cell carcinoma [13]. UCA1 includes three exons totaling $1.4 \mathrm{~kb}$ in length. In bladder transitional cell carcinoma, UCA1 is found to be significantly overexpressed and functions as a biomarker for the diagnosis of bladder cancer. Subsequently, another isoform ( $2.2 \mathrm{~kb})$ was identified as the cancer upregulated drug-resistant (CUDR) gene in the doxorubicin-resistant subline of human squamous carcinoma A431 cells [14]. Although these studies suggest an oncogenic role of UCA1 in bladder cancer, the underlying mechanism is largely unknown. A recent report shows that UCA1 along with hnRNP I inhibits p27 protein expression by competitive inhibition. Moreover, UCA1 has an oncogenic role in breast cancer both in vitro and in vivo [10].

In this study, we explored a possiblerole of IncRNA UCA1 in the development of ischemia/ reperfusion (I/R)-induced injury. Our results show that UCA1 contributes to the apoptosis of cardiomyocytes mediated by caspase3 through down-regulation of the tumor suppressor gene $\mathrm{p} 27$. Our finding provides new insight into the roles of lncRNAs in the development of ischemia/reperfusion (I/R)-induced injury.

\section{Materials and Methods}

Myocardial ischemia/reperfusion injury in Sprague-Dawley rats

Adult male Sprague-Dawley rats (220-230 g), provided by the Animal Facility, Health Science Center of Peking University (Beijing, China), were housed in the laboratory animal room and maintained at $25^{\circ} \mathrm{C} \pm 1^{\circ} \mathrm{C}$ with $65 \% \pm 5 \%$ humidity on a $12 \mathrm{~h}$ light/dark cycle (lights on from 07:30 to 19:30) for at least 1 week before the experiments. Animals were given food and water ad libitum. All experimental protocols described in this study were approved by the Ethics Review Committee for Animal Experimentation of Harbin Medical 
University. Rats underwent 30 min myocardial ischemia via left anterior descending artery occlusion followed by $24 \mathrm{~h}$ reperfusion as previously described [15]. Briefly, rats were anesthetized with sodium pentobarbital $(90 \mathrm{mg} / \mathrm{kg})$. A lateral thoracotomy $(1.5-\mathrm{cm}$ incision between the second and third ribs) was performed to provide exposure of the LAD, while avoiding rib and sternal resection, retraction, and rotation of the heart. Vascular bundles in the vicinity were coagulated by use of a microcoagulator (Medical Industries). An 8-0 nylon suture was placed around the LAD at 2 to $3 \mathrm{~mm}$ from the tip of the left auricle, and a piece of soft silicon tubing ( $0.64 \mathrm{~mm} I D, 1.19 \mathrm{~mm}$ OD) was placed over the artery. All rats were subjected to a 30-minute coronary occlusion by tightening and tying the suture. After 24 hours of reperfusion, the aorta was cannulated, and the hearts were quick frozen in liquid $\mathrm{N}_{2}$ for further experiments.

\section{2,3,5-triphenyltetrazolium chloride (TTC) staining}

After reperfusion, the rats were deeply anesthetized with 3.5\% chloral hydrate and then decapitated, after which their whole hearts were rapidly removed. Coronal sections ( $\mathrm{n}=10$ for each group) were cut into $2-\mu \mathrm{m}$ slices and stained with standard 2\% 2,3,5-triphenyltetrazolium chloride (Sigma, St. Louis, MO, USA) for $10 \mathrm{~min}$ at $37^{\circ} \mathrm{C}$ followed by overnight immersion in $4 \%$ formalin. Infarct volume, expressed as a percentage of whole-heart volume, was measured by an image processing and analysis system $(1.25 \times$ objective, Q570IW; Leica, Wetzlar, Germany) and was calculated by integration of the infarct area on each heart section along the rostralcaudal axis. Quantitative analysis of heart section was performed by capturing images with a Nikon D80. The data were analyzed with Image Pro-Plus-6 software.

\section{Cardiomyocyte culture and treatment}

Cardiomyocytes were isolated from 1-2-day-old Sprague-Dawley rats as we previously described [16]. Briefly, after dissected hearts were washed, they were minced in HEPES-buffered saline solution. Tissues were then dispersed through a series of incubations at $37^{\circ} \mathrm{C}$ in HEPES-buffered saline solution containing $1.2 \mathrm{mg} / \mathrm{ml}$ pancreatin and $0.14 \mathrm{mg} / \mathrm{ml}$ collagenase (Worthington, Lakewood, NJ, USA). After centrifugation, cells were resuspended in Dulbecco's modified Eagle medium/F-12 (GIBCO, Grand Island, NY, USA) containing 5\% heat-inactivated horse serum, $0.1 \mathrm{mM}$ ascorbate, insulin-transferring-sodium selenite media supplement (Sigma, St. Louis, MO, USA), $100 \mathrm{U} / \mathrm{ml}$ penicillin, $100 \mathrm{mg} / \mathrm{ml}$ streptomycin and $0.1 \mathrm{mM}$ bromodeoxyuridine. The dissociated cells were preplated at $37^{\circ} \mathrm{C}$ for $1 \mathrm{~h}$. The cells were then diluted to $1 \times 10^{6}$ cells $/ \mathrm{ml}$ and 3 to $5 \times 10^{3}$ cells $/ \mathrm{cm}^{2}$ were seeded in various $10 \mathrm{mg} / \mathrm{ml} \mathrm{laminin}$-coated culture dishes according to the specific experimental requirements. And the medium volume was $200 \mu \mathrm{L} / \mathrm{cm}^{2}$. Cells were treated with $200 \mu \mathrm{M}$ or $500 \mathrm{mM} \mathrm{H}_{2} \mathrm{O}_{2}$ except when indicated otherwise. 5 .

\section{Microarray Analysis for IncRNAs}

Kangchen Bio-tech (Shanghai PR China) performed the microarray work in which 10 samples (5 samples for the reperfusion group and 5 for the sham group) were used for IncRNA microarray analysis. Total RNA from each sample was quantified using the NanoDrop ND-1000 and RNA integrity was assessed using standard denaturing agarose gel electrophoresis. For microarray analysis, the Agilent Array platform was employed. Sample preparation and microarray hybridization were performed based on the manufacturer's standard protocols with minor modifications. Briefly, mRNA was purified from total RNA after removal of rRNA (mRNA-ONLY ${ }^{\mathrm{TM}}$ Eukaryotic mRNA Isolation Kit, Epicentre). Then, each sample was amplified and transcribed into fluorescent cRNA along the entire length of the transcripts without 3 ' bias utilizing a random priming method. The labeled cRNAs were hybridized onto the rat IncRNA Array v2.0 (8 $\times$ $60 \mathrm{~K}$, Arraystar). After washing the slides, the arrays were scanned by the Agilent Scanner G2505C.

Total RNA Isolation, Reverse Transcription-PCR, and Real-time PCR

Total RNA was isolated using TRIzol reagent (Invitrogen), and cDNA was synthesized with PrimeScript reverse transcriptase (TaKaRa, Dalian, China) and oligo(dT) (20 bp) following the manufacturer's instructions. Reverse transcription PCR (RT-PCR) or real-time PCR was performed to analyze mRNA expression. The RT-PCR program was as follows: $94{ }^{\circ} \mathrm{C}$ for $5 \mathrm{~min}$, followed by 35 cycles of $94^{\circ} \mathrm{C}$ for $30 \mathrm{~s}, 50$ ${ }^{\circ} \mathrm{C}$ annealing for $30 \mathrm{~s}$, and $72{ }^{\circ} \mathrm{C}$ for $30 \mathrm{~s}$. Real-time PCR was performed using SYBR Premix Ex TaqTM II kit (TaKaRa, Dalian, China). The real-time PCR program was as follows: $94^{\circ} \mathrm{C}$ for $10 \mathrm{~s}$, followed by 40 cycles of $94{ }^{\circ} \mathrm{C}$ for $5 \mathrm{~s}, 52^{\circ} \mathrm{C}$ for $30 \mathrm{~s}$ to anneal, and $72{ }^{\circ} \mathrm{C}$ for $15 \mathrm{~s}$. The relative level of UCA1 was determined using the $2^{\text {-detta delta Ct }}$ analysis method [17]. 
The primers used for UCA1 and GAPDH were listed as follows: UCA1-RT-F: TTT GCC AGC CTC AGC TTA AT; UCA1-RT-R: TTG TCC CCA TTT TCC ATC AT. GAPDH-RT-F: CCA CCC ATG GCA AAT TCC ATG GCA; GAPDHRT-R: TCT AGA CGG CAG GTC AGG TCC ACC.

Western Blot Analysis

Proteins were extracted from the primary cardiomyocytes in RIPA buffer (1\% Triton X-100, 150 $\mathrm{mmol} / \mathrm{L} \mathrm{NaCl}, 5 \mathrm{mmol} / \mathrm{L}$ EDTA, and $10 \mathrm{mmol} / \mathrm{L}$ Tris-HCl (pH 7.0)) (Solarbio, China) supplemented with a protease inhibitor cocktail (Cat: I3786-1ML, Sigma, St. Louis, MO, USA). The cell lysates were separated by $10 \%$ SDS-PAGE and transferred electrophoretically to a PVDF membrane (Millipore Corporation, Billerica, MA, USA). After soaking with $8 \%$ milk in PBS (pH 7.5), the membranes were incubated with the following specific primary antibodies: anti-p27 (sc-528) (Santa Cruz Biotechnology, Dallas, TX, USA), anti-bcl-2 (sc492) (Santa Cruz Biotechnology, Dallas, TX, USA), anti-caspase3 (ab4051) (Abcam, England) and anti- $\beta$ actin (sc-8432) (Santa Cruz Biotechnology, Dallas, TX, USA). After incubation overnight, the appropriate HRP-conjugated anti-rabbit IgG secondary antibodies (Abmart, all at a 1:5000) were subsequently applied, and immunodetection was achieved using the ECL Plus detection system (Millipore Corporation, Billerica, MA, USA) according to the manufacturer's instructions. GAPDH was used as the internal control.

\section{Plasmid construction and lentivirus production}

In the PCR reactions for cloning purposes, we used Phusion enzyme (Thermo Fisher Scientific, Pittsburgh, PA, USA). The entire UCA1 CDNA (1.4 kb isoform) was amplified by RT-PCR using primers UCA1-BamHI-F (GCG GAT CCT TTA TCA GGC ATA TTA GCT TTA A) and UCA1-EcoRI-R (GCG AAT TCT GAC ATT CTT CTG GAC AAT G) and then cloned into the expression vector PCDH-CMV-MSC-EF1-copGFP (System Biosciences) using a Cold Fusion kit (System Biosciences). The restriction sites were underlined. Recombinant lentivirus was generated from 293T cells using calcium phosphate precipitation. Primary cardiomyocytes were transfected with lentivirus using polybrene $(8 \mathrm{ug} / \mathrm{ml})$.

\section{Transfection of siRNAs}

Small interfering RNAs (siRNAs) targeting murine UCA1 mRNA (si-UCA1) and negative control siRNA (si-Ctrl) were synthesized by RiboBio (Guangzhou, China). siRNAs against TUC338 were designed with siDESIGN (http://www.dharmacon.com/designcenter/designcenterpage.aspx), and the two highestranked target sequences were synthesized [17].

Transfections were performed in 6-, 24-, or 96-well plates after seeded cells were cultured for $24 \mathrm{~h}$. siRNA reagents were transfected using HiPerFect transfection reagent (QIAGEN, Germany) according to the manufacturer's protocol [18]. Briefly, $5 \times 10^{3}$ cells $/ \mathrm{cm}^{2}$ were seeded in $200 \mu \mathrm{L} / \mathrm{cm}^{2}$ culture. siRNAs or pcDNAs were pre-incubated with HiPerFect transfection reagent at room temperature for $10 \mathrm{~min}$. The complex was then transfected into the cardiomyocytes cells at a final concentration of $50 \mathrm{nM}$. The transfected cells were incubated under normal growth conditions for $48 \mathrm{~h} . \%$ of transfection is deined as the $\%$ of GFP labelled cells over Hoechst.

UCA1 siRNA and control siRNA sequences were as follows:

UCA1 siRNA: 1. 5'-CGA GAC GUC CAU CGA CAU TT-3'; 2. 5'-GUC CAU CGA UGG ACG UCU CG-3'; 3. 5'-CCC TGT AAC GCT CAA GTT AGG-3'; 4. 5'-CAG TTC CCT AAC TTG AGC GTT A-3'; Control siRNA: 5'-UUC UCC GAA CGU GUC ACG UTT-3'.

Dimethyl thiazolyl diphenyl tetrazolium (MTT) assay

To explore the effect of UCA1 on cell viability, 5,000 cells per well in a $100 \mu \mathrm{l}$ medium were seeded in 96-well plates. At 70-80\% confluence, the cells were transfected with pUCA1 (50 nM) / pcDNA (50 nM) or siRNA-UCA1 / siRNA-control (50 nM). Every $24 \mathrm{~h}$ after transfection, $20 \mu \mathrm{l}$ of the 3-(4,5-dimethylthiazol-2yl)-2,5-diphenyltetrazolium bromide (MTT) reagent (Solarbio, China) was added to wells and incubated with the cells for $4 \mathrm{~h}$. After removing the medium, the blue formazan was dissolved with $200 \mu$ ldimethyl sulfoxide (DMSO) (Sigma, St. Louis, MO, USA), and absorbance was measured at $550 \mathrm{~nm}$. Wells containing only cardiomyocytes cells served as blanks. 


\section{Cellular Physiology Cell Physiol Biochem 2015;35:1986-1998

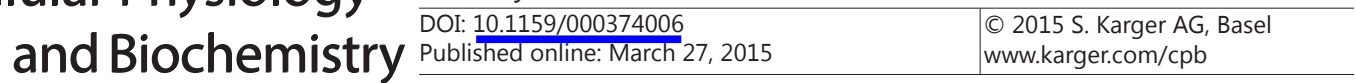

Liu et al.: Reduced UCA1 Level Leads to Cardiomyocyte Apoptosis

\section{Determination of ROS}

To evaluate tissue production of ROS $\left(\mathrm{O}_{2}{ }^{-}\right.$in particular), fresh, frozen left ventricular myocardium (7$\mu \mathrm{m}$ sections) was incubated with dihydroethidium (DHE) (Vigorous Biotechnology Beijing Co., Ltd) (with final concentration of $5 \mu \mathrm{M}$ ) for 1 hour at room temperature. Following 3 washes by PBS, the sections were stained with Hoechst $33258(10 \mu \mathrm{g} / \mathrm{ml})$ for 5 minutes at room temperature. Slides were rinsed 3 times with PBS, mounted with prolong gold antifade mounting reagent and coverslipped. Tissue slideswere examined using fluorescence microscope. The numbers of DHE-positive nuclei and the total nuclei were counted (50 fields per group), as described previously [19].

\section{Immunohistochemistry}

Rats were sacrificed 24 and $72 \mathrm{~h}$ after underlying ischemia/reperfusion (I/R)-induced injury with an overdose of $3.5 \%$ chloral hydrate and transcardially perfused with $0.9 \%$ saline solution followed by $4 \%$ ice-cold phosphate-buffered paraformaldehyde (PFA). Hearts were removed, postfixed overnight, and equilibrated in phosphate-buffered $30 \%$ sucrose. Coronal sections at 1.0 to $2.0 \mathrm{~mm}$ from the bregma were cut with a cryostat (Leica CM3000, Leica) to a thickness of $25 \mu \mathrm{m}$ and used for immunohistochemical staining.

Frozen sections were double-stained by phenotypic markers using rabbit polyclonal anti-c-caspase 3 antibody (1:100; ab2302, Abcam, England) as the primary antibody at $37^{\circ} \mathrm{C}$ for $60 \mathrm{~min}$ or at $4^{\circ} \mathrm{C}$ overnight and then labeled with HRP-conjugated anti-rabbit IgG at $37^{\circ} \mathrm{C}$ for $60 \mathrm{~min}$. Next, the coverslips were mounted with DABCO and analyzed by upright microscope (Carl Zeiss). The numbers of c-caspase3-positive cells and the total cells were counted (50 fields per group).

\section{Immunofluorescence}

Primary cardiomyocytes were cultured on 6-well chamber slides and fixed with 4\% paraformaldehyde for $10 \mathrm{~min}$ at $-20^{\circ} \mathrm{C}$. The slides were washed in PBS for $5 \mathrm{~min}$ per time (three times) and were then incubated with a polyclonal antibody against p27 (1:50 diluted in PBS with 1\% BSA) for $2 \mathrm{~h}$ at room temperature. After washing with PBS for three times (5 min per time), the slides were incubated with FITC-conjugated anti-mouse IgG (1:100 diluted in PBS with 1\% BSA) for $1 \mathrm{~h}$ at room temperature. Three times after washing the slides in PBS, the slides were incubated with Hoechst $33258(10 \mu \mathrm{g} / \mathrm{ml})$ for $5 \mathrm{~min}$. Then, the slides were washed again and examined using a fluorescence microscope. The quantification of FITC intensity was carried out with NIH ImageJ software [20].

\section{Hoechst 33258 staining}

Primary cardiomyocytes were cultured in 6-well plates. After $48 \mathrm{~h}$ transfection with siRNA targeting UCA1 or negative control, cells were washed with PBS and stained with Hoechst $33258(10 \mu \mathrm{g} / \mathrm{ml})$ for $5 \mathrm{~min}$ before being washed three times with PBS. Cells were visualized by a Leica inverted microscope at 100x magnification. Hoechst-positive cardiomyocytes were carefully evaluated under double-blind conditions. At least 3 visual fields were chosen for analysis. The rate of apoptotic cell nuclei is deined as apoptotic positive cell nuclei/total cell nuclei in the field.

\section{Quantification of apoptotic cells}

To quantify apoptotic cells, flow cytometry was performed with an Annexin V-fluorescein-5isothiocyanate apoptosis detection kit (Bio-vision, USA). $48 \mathrm{~h}$ after transfection with pcUCA1 (50 nM) and pcDNA (50 nM), cardiomyocytes were harvested in a $5 \mathrm{ml}$ tube. Then, the cells were washed with cold PBS and resuspended in 1×binding buffer (10 mM HEPES, $140 \mathrm{mM} \mathrm{NaCl}, 2.5 \mathrm{mM} \mathrm{CaCl} 2, \mathrm{pH} 7.4)$ at a final concentration of $1 \times 10^{6}$ cells $/ \mathrm{ml}$. FITC-AnnexinV $(5 \mu \mathrm{l})$ and propidium iodide were gently mixed and incubated with the cells for $15 \mathrm{~min}$ at a room temperature. After incubation, the samples were analyzed by flow cytometry within $1 \mathrm{~h}$.

\section{Statistical analysis}

Data were presented as mean \pm SE. Differences between groups were compaired by a one-way ANOVA method using Graphpad prism. $P<0.05$ was considered as statistically significant difference. 


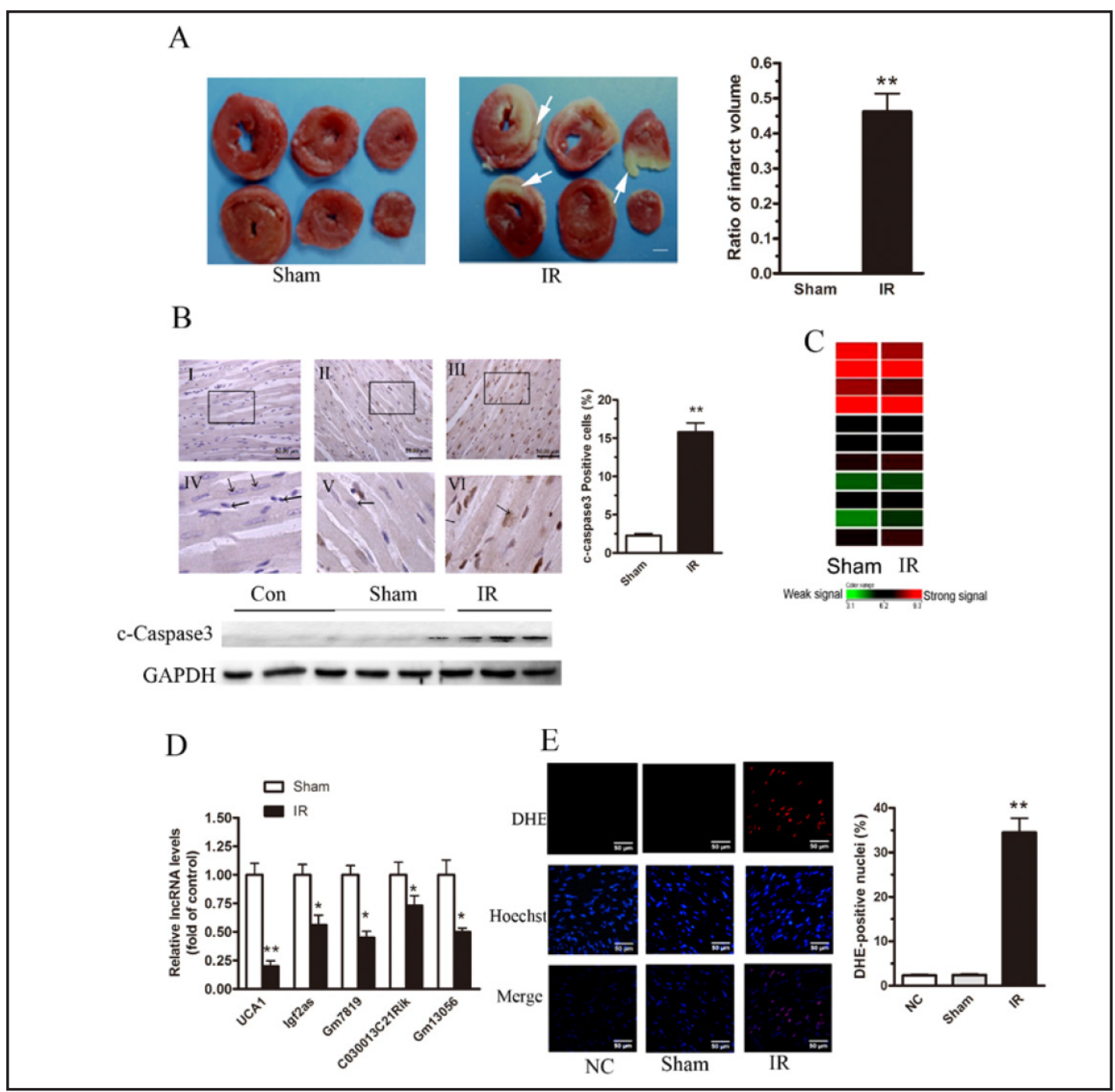

Fig. 1. The cardiac ischemia/reperfusion injury model was accompanied by reduced UCA1 expression. (A) Statistical analysis of cardiac injury indicated by TTC staining. The images shown here are representative samples of 3 independent rat hearts. White arrows indicate the infarct volume and the bar equals to 1 mm. (B) Immunohistochemistry for c-caspase3 in cardiomyocytes after myocardial I/R injury. I represents negative control group, II represents sham group, III represent IR group, the black lined-boxes (in I, II, and III)were shown by high-magniication micrographs $(1000 \times)$ of VI,VI, and VI, respectively. Arrows show the nuclei of blood. (C) lncRNA microarray was applied to determine the changed lncRNA expression profile after I/R in rats. (D) The results of IncRNA microarray were verified by qPCR. (E) ROS production in hearts of rats from sham group or IR group. Frozen myocardial tissue sections were stained with DHE (red), and nucleus with Hoechst (blue). Data were from 3 independent experiments each with 3 rats per. The percentage of DHE-positive nuclei was quantified (50 fields from 3 rats per group, scale bar=50 $\mu \mathrm{m}$ ). $\mathrm{n}=3$, ${ }^{*} \mathrm{P}<$ $0.05 ; * * \mathrm{P}<0.01$ versus control.

\section{Results}

Cardiac ischemia/reperfusion injury rat model is accompanied by reduced UCA1 expression

To determine the potential role of IncRNAs in ischemia/reperfusion injury, we generated a partial cardiac ischemia/reperfusion model in rats. After ischemia/reperfusion treatment, TTC staining assays revealed that heart tissue was significantly injured with obvious infarct volume (Fig. 1A). Moreover, immunohistochemistry staining revealed the presence 

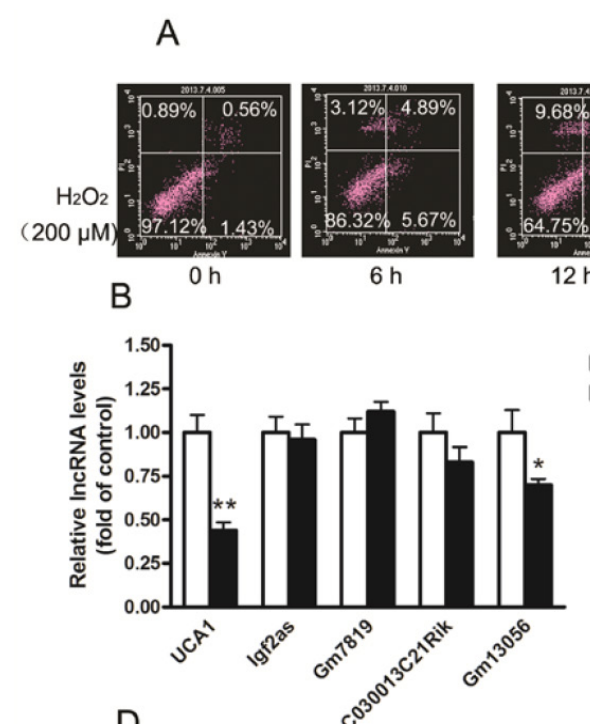

D

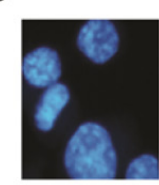

Con

$\mathrm{F}$

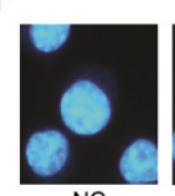

NC
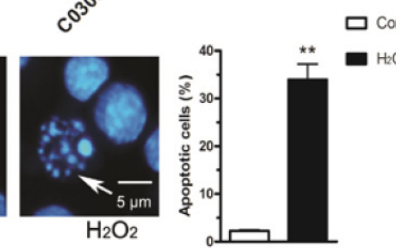

口 con

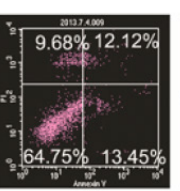

$12 \mathrm{~h}$

$\square$ Con

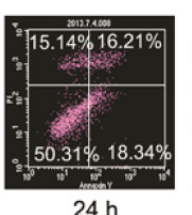

$24 \mathrm{~h}$

C
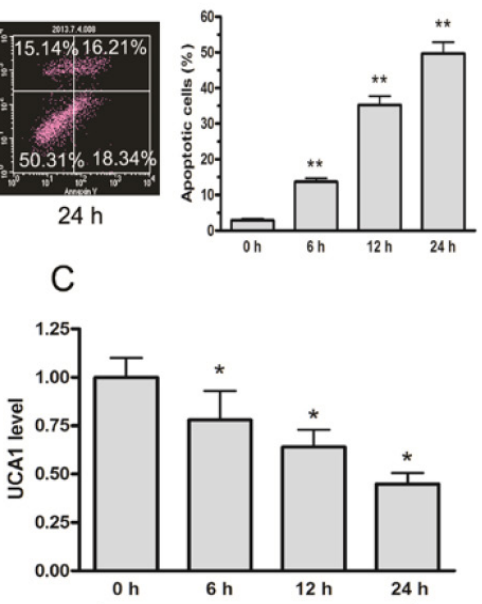

$\mathrm{H}_{2} \mathrm{O}_{2}(200 \mu \mathrm{M}) \quad$ Time (h)

$\mathrm{E}$

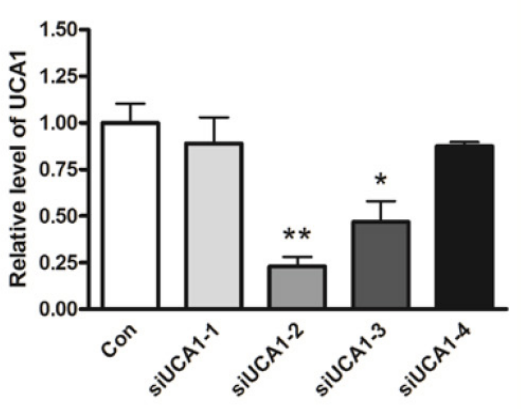

Fig. 2. UCA1 expression levels inversely correlates with $\mathrm{H}_{2} \mathrm{O}_{2}$-induced apoptosis in cardiomyocytes. (A) Cardiomyocytes were treated with $200 \mathrm{\mu M} \mathrm{H}_{2} \mathrm{O}_{2}$ for the indicated times. Apoptotic cells were analyzed by flow cytometry analysis of the active caspase-3 using the Vybrant FAM caspase-3 assay. (B) RT-PCR analysis of IncRNA expression levels upon $\mathrm{H}_{2} \mathrm{O}_{2}$ treatment. (C) $\mathrm{H}_{2} \mathrm{O}_{2}$ treatment reduced UCA1 levels also in a time-dependent manner. Cardiomyocytes were treated with $\mathrm{H}_{2} \mathrm{O}_{2}$ at the indicated times, and the expression of UCA1 was analyzed; ${ }^{*} \mathrm{P}<0.05$ versus control. (D) Cardiomyocytes were treated with $\mathrm{H}_{2} \mathrm{O}_{2}$ for $24 \mathrm{~h}$, and cell apoptosis was determined using Hoechst staining. (E) siRNA targeting UCA1 were applied to inhibit the level of UCA1 and qPCR was used to quantify the relative level of UCA1. (F) Knockdown of UCA1 reduces apoptotic responses induced by $\mathrm{H}_{2} \mathrm{O}_{2}$. Cardiomyocytes were transfected with siUCA1 or NC. Twenty-four hours after transfection, cells were treated with $\mathrm{H}_{2} \mathrm{O}_{2} . \mathrm{n}=3$, ${ }^{*} \mathrm{P}<0.05$; ** $\mathrm{P}<0.01$ versus control.

of c-caspase3 in the heart sections after I/R compared with the sham group (Fig. 1B). To profile the expression of IncRNAs after I/R, lncRNA microarray was applied and the result of lncRNA microarray showed UCA1 was increased the most after I/R in rats (Fig. 1C). We then selected the most significantly downregulated five lncRNAs and validated the results using qPCR. As shown in Fig. 1D, UCA1 was strongly inhibited compared to the other lncRNAs, and this difference was highly significant. To understand the pathophysiological role of UCA1, ROS contents were also determined using DHE staining. As shown in Fig. 1E, the ROS level was significantly enhanced in the I/R injury hearts, consistent with the decreased UCA1 expression.

UCA1 expression levels inversely correlates with H2O2-induced apoptosis in cardiomyocytes Hydrogen peroxide $\left(\mathrm{H}_{2} \mathrm{O}_{2}\right)$ is well known to be important factor for inducing cell death. We tested the effect of $\mathrm{H}_{2} \mathrm{O}_{2}$ on cardiomyocytes and observed that treatment with $200 \mu \mathrm{M}$ 
A

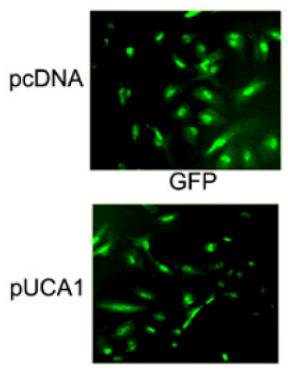

GFP
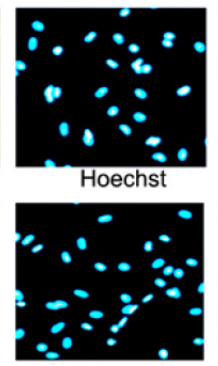

Hoechst
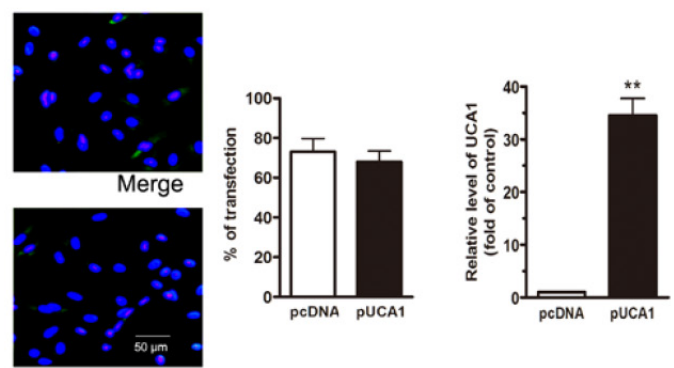

Merge

B pcDNA pUCA1

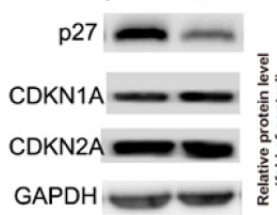

D

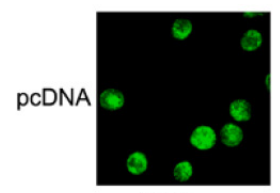

FITC

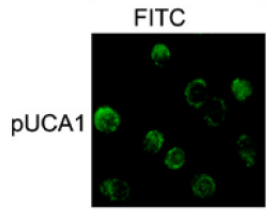

FITC

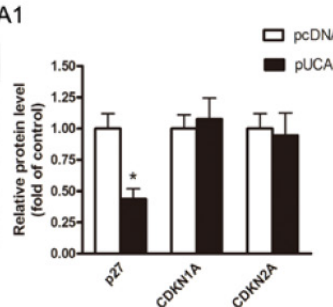

ㅁ PCDNA

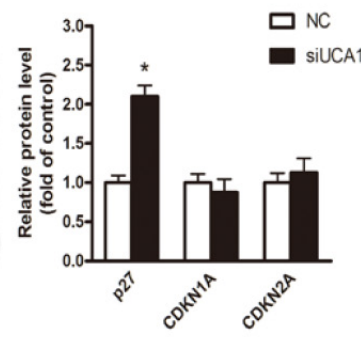

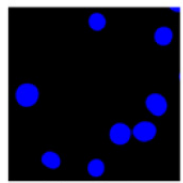

Hoechst

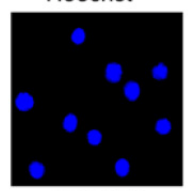

Hoechst

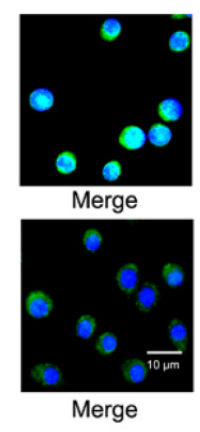

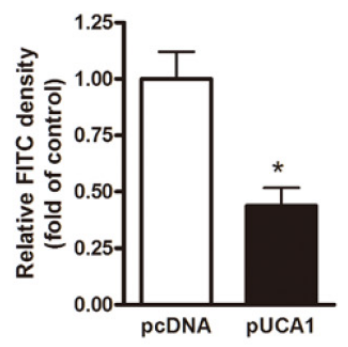

Fig. 3. UCA1 suppresses p27 protein levels. (A) pcUCA1 or pcDNA was transfected into primary cardiomycytes and the relative level of UCA1 was determined using RT-qPCR. . Scale bar represents $50 \mu \mathrm{m}$. (B) Transfection of pUCA1 significantly suppresses p27 expression. (C) Knockdown of UCA1 enhances the protein level of p27 compared with control siRNA. (D) Suppression of the p27 protein by ectopic expression of UCA1 using an immunofluorescence assay. Scale bar represents $10 \mu \mathrm{m}$. Error bars represent S.E.M., $\mathrm{n}=3$. $* \mathrm{P}<$ $0.05 ;{ }^{* *} \mathrm{P}<0.01$ versus control.

$\mathrm{H}_{2} \mathrm{O}_{2}$ trigged apoptosis using flow cytometry. As shown in Fig. 2A, treatment with $200 \mu \mathrm{M} \mathrm{H}_{2} \mathrm{O}_{2}$ significantly enhanced apoptotic cells in a time-dependent manner. To determine whether IncRNAs are involved in the apoptosis pathway induced by $\mathrm{H}_{2} \mathrm{O}_{2}$, qPCR was applied to detect lncRNAs in response to $\mathrm{H}_{2} \mathrm{O}_{2}$ treatment (Fig. 2B). As shown in Fig. 2B, UCA1 was more repressed compared with other four lncRNAs upon $\mathrm{H}_{2} \mathrm{O}_{2}$ treatment for $24 \mathrm{~h}$. Furthermore, $\mathrm{H}_{2} \mathrm{O}_{2}$ treatment reduced UCA1 levels also in a time-dependent manner (Fig. 2C). Next, we investigated the function of UCA1 in the apoptosis machinery. Upon exposure to $200 \mu \mathrm{M}$ $\mathrm{H}_{2} \mathrm{O}_{2}$ for $24 \mathrm{~h}$, cardiomyocytes displayed obvious apoptotic morphological characteristics (Fig. 2D). To explore the effects of UCA1 on cell apoptosis, four siRNA targeting UCA1 were applied and the mRNA levels of UCA1 were tested using qPCR. As shown in Fig. 2E, siRNA-2 targeting UCA1 demonstrated the most obvious silencing effect with over $75 \%$ reduction of UCA1. Thus, siRNA-2 was selected for the further study. Moreover, knockdown of UCA1 by siRNA but not the negative control (NC) also enhanced apoptotic morphological hallmarks, as confirmed by Hoechst staining (Fig. 2F). These data suggest that UCA1 expression levels inverselyinversely correlates with $\mathrm{H}_{2} \mathrm{O}_{2}$-induced apoptosis in cardiomyocytes and has a functional role in the response of cardiomyocytes to injury. 
Fig. 4. Knockdown of UCA1 reduces cell growth and prompts cell apoptosis. Cardiomyocytes were transfected with pUCA1 or pcDNA for 24, 48 , or $72 \mathrm{~h}$. Upregulation of UCA1 enhanced cell viability in primary cardiomyocytes by $20 \%$ and $30 \%$ at $48 \mathrm{~h}$ and $72 \mathrm{~h}$, respectively (A), whereas downregulation of UCA1 decreased cell viability by $25 \%$ and $30 \%$ at $48 \mathrm{~h}$ and $72 \mathrm{~h}$, respectively (B). (C) Inhibition of UCA1 enhanced cell apoptosis by nearly fourfold versus the negative control in primary cardiomyocytes as tested using an Annexin V and PI kit. (D) The number of apoptotic cells increased in the primary cardiomyocytes transfected with siRNA targeting UCA1, as examined by Hoechst 33342 staining. The white arrow indicates apoptotic cells. Error bars represent S.E.M., $\mathrm{n}=3 .{ }^{*} \mathrm{P}<0.05 ;{ }^{*} \mathrm{P}<0.01$ versus control.

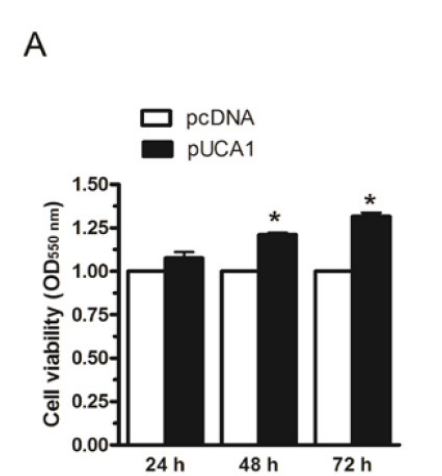

B
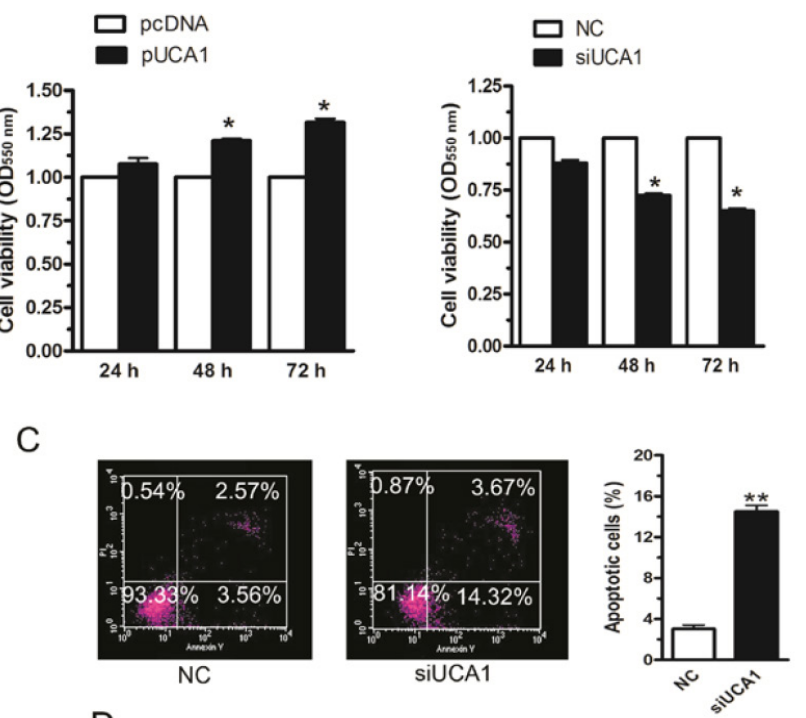

D
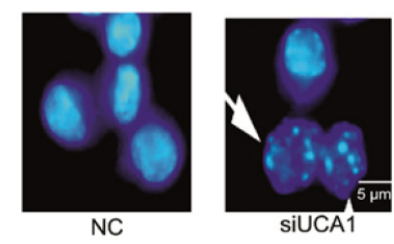

UCA1 suppresses $p 27$ protein levels

UCA1 has been previously reported to inhibit the expression of p27 [10]. Thus, we validated the regulatory effect of UCA1 on the level of the tumor suppressor p27. First of all, pcUCA1 or pcDNA was transfected into primary cardiomycytes and the relative fluorescence level of GFP marker was quantified using a fluorescence microscope. As shown in Fig. 3A, over $90 \%$ of cells were effectively transfected with pcUCA1 or pcDNA. Next, the level of UCA1 in cells transfected with pUCA1 or pcDNA was determined. As shown in Fig. 3A, UCA1 was overexpressed more 34-fold compared with pcDNA. Then, western blot revealed that transfection of pcUCA1 significantly reduced p27 protein levels, while no obvious change of CDKN1A and CDKN2A was defined (Fig. 3B). In comparison, knockdown of UCA1 resulted in higher p27 protein levels without changes in CDKN1A and CDKN2A expression pattern (Fig. 3C). Consistent with this finding, immunofluorescence assays for p27 revealed significant suppression by UCA1 (Fig. 3D), suggesting that UCA1 directly inhibits p27 at the protein level.

Knockdown of UCA1 reduces cell viability and prompts cell apoptosis

In order to determine the effect of UCA1 on cell viability, cardiomyocytes were transfected with pUCA1 or pcDNA for 24,48 , or $72 \mathrm{~h}$. As shown in Fig. $4 \mathrm{~A}$, upregulation of UCA1 enhanced cell viability in the primary cardiomyocytes by $20 \%$ and $30 \%$ at $48 \mathrm{~h}$ and $72 \mathrm{~h}$, respectively, whereas downregulation of UCA1 decreased cell viability by $25 \%$ and $30 \%$ at $48 \mathrm{~h}$ and $72 \mathrm{~h}$, respectively (Fig. 4B). We next observed the effect of UCA1 on cell apoptosis. The results showed that inhibition of UCA1 enhanced cell apoptosis by nearly fourfold versus the negative control in primary cardiomyocytes $(14.32 \% \pm 0.78$ vs. $3.56 \% \pm$ 0.32, Fig. 4C). Morphology was also examined by Hoechst 33342 staining. As shown in Fig. 4D, the number of apoptotic cells increased in the primary cardiomyocytes transfected with siRNA targeting UCA1. These data suggest that UCA1 can modulate primary cardiomyocyte viability and that downregulation of UCA1 induces primary cardiomyocytes apoptosis. 
Fig. 5. Upregulation of p27 expression contributed to UCA1 downregulation-related cardiomyocyte apoptosis. (A) Ectopic expression of p27 significantly induced cell apoptosis with enhanced caspase 3 activation, which then reduced Bcl-2 protein levels. (B) Using a specific siRNA targeting p27, caspase3 activation was significantly reduced, and the Bcl-2 protein level was enhanced. (C) Overexpression of p27 also significantly enhanced primary cardiomyocyte apoptosis as determined using flow cytometry analysis. (D) Inhibition of p27 reduced cariomyocyte apopotosis using flow cytometry analysis. Error bars represent S.E.M., $\mathrm{n}=3$. ${ }^{*} \mathrm{P}$ $<0.05$; ${ }^{* *} \mathrm{P}<0.01$ versus control.
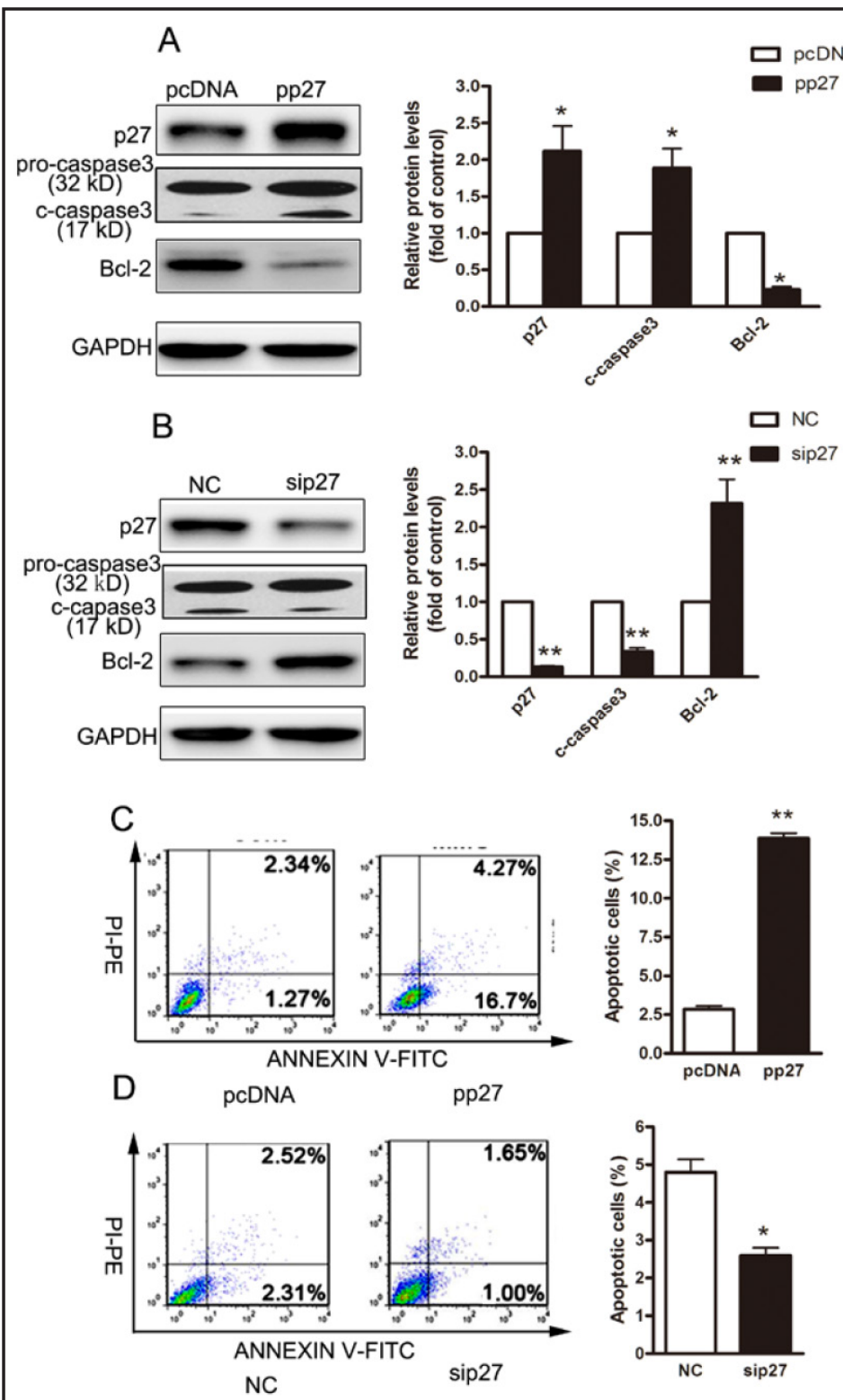

Fig. 6. Summary. ROS production reduced the expression of IncRNA UCA1 and induced primary cardiomyocytes apoptosis partially through stimulation of $\mathrm{p} 27$ protein expression.

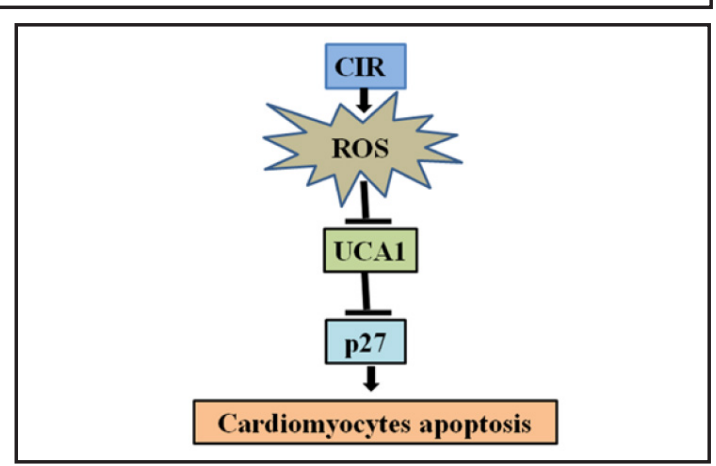

Overexpression of p27 induces caspase 3 activation in primary cardiomyocytes

To further investigate the mechanisms underlying the functional effects of p27 on cell apoptosis, we over-expressed p27 in primary cardiomyocytes. A p27 expression vector was transiently transfected into primary cardiomyocytes, resulting in a dramatic increase in p27 levels with respect to empty vector-transfected cells (Fig. 4A). Furthermore, we also 
found that overexpression of p27 significantly induced cell apoptosis through enhanced caspase 3 activation, which then decreased Bcl-2 protein levels. In addition, when p27 was silenced by a siRNA, caspase 3 activation was significantly reduced and Bcl-2 protein levels were increased (Fig. 5B). Meanwhile, overexpression of p27 was also found to significantly enhanced primary cardiomyocyte apoptosis using flow cytometry analysis (Fig. 5C). Vice verse, cardiomyocytes apoptosis was obviously reduced when the level of p27 was inhibited (Fig. 5D). These results indicate that overexpression of $\mathrm{p} 27$ expression contributes to UCA1 downregulation-related cardiomyocyte apoptosis.

\section{Discussion}

lncRNAs have emerged as important players in pathology processes [21]. Increasing evidence suggests that lncRNAs may function as pro-apoptotic or anti-apoptotic regulators [22]. They are frequently dysregulated in various disease models [23]. In support of this notion, we show that UCA1 is downregulated in I/R-induced heart injury and that its expression is negatively correlated with the tumor suppressor p27. Furthermore, we also demonstrate that ectopic expression of UCA1 promotes cell survival, while UCA1-siRNA suppresses cell viability and enhances cardiomyocyte apoptosis in vitro. Importantly, our results suggest that this UCA1-mediated cell apoptosis contributes to cardiac injury partially through enhanced p27 protein levels. Thus, our study provides supporting evidence that UCA1 has an important role in ischemic cardiac injury.

Cardiovascular disease is a leading cause of hospitalization and death worldwide [24]. Thus, elucidating molecular mechanisms and discovering impactful therapeutic targets are especially important for the treatment of cardiac injury. Our present work demonstrates that $\mathrm{H}_{2} \mathrm{O}_{2}$-treated cardiomyocytes exhibited significantly reduced UCA1 levels. In searching for the effect of I/R-induced injury on UCA1 level, we found that the UCA1 level was reduced in the ischemic heart after reperfusion. Downregulation of UCA1 enhanced cardiac apoptosis through enhanced p27 protein expression. Furthermore, overexpression of p27 suppressed cardiomyocyte viability and prompted cell apoptosis. Our results reveal a novel mechanism for the regulation of cardiomyocyte injury by lncRNA UCA1 (Fig. 6).

The fate of cardiac cells after ischemic injury is determined by the balance between cell survival and death [25]. Apoptosis is regarded as one cell death mechanisms and can be induced by various stress conditions, such as oxidative stress and endoplasmic reticulum stress. Following myocardial ischemia/reperfusion injury, enhanced apoptosis has been demonstrated [26]. Studies have indicated that upregulation of p27 significantly enhances cell apoptosis [27].In previous studies, regulation of p27 has been reported at different levels [10]. Alterations of cell cycle regulatory molecules play key roles in various diseases, such as cancer and cardiac injury [28]. The Cdk inhibitor p27KIP1 (p27), which negatively controls cell-cycle progression, has been observed to be dysregulated in different cell types [29]. In this study, we determined the levels of p27 protein in heart injury after I/R. Indeed, p27 upregulation in heart injury has been associated with enhanced ROS production and increased cell apoptosis. Meanwhile, a marked reduction in the abundance of UCA1 is found in cardiac injury after I/R. In previous studies, p27 has been identified as a target of UCA1 [10]. In this study, we validated that reduction of UCA1 levels plays a pro-apoptotic role in primary cultured cardiomyocytes, in part through stimulation of p27 protein expression. These results are in agreement with the observed levels of UCA1, p27 and apoptosis after cardiac I/R injury, suggesting that UCA1 might have an important role during I/R injury.

p27 is known primarily as a suppressor of cellular proliferation and our studies strongly indicate such a proapoptotic function for $\mathrm{p} 27$ [30]. We observed the proapoptotic function of p27 the cardiomyocytes model in vitro. The reduced UCA1 level led to increased protein expression of p27 and resulted in the subsequent activation of caspase 3 in cardiomyocytes. When p27 was overexpressed by an ectopic plasmid, increased expression of cleaved caspase-3 was observed. We propose that enhanced cleaved caspase-3 in cardiomyocytes 
are linked to a significant cell apoptosis. The precise molecular mechanisms underlying the cardiomyocyte apoptosis may involve UCA1 downregulation-related p27 overexpression. Taken together, our results demonstrate that reduction of UCA1 levels serves a proapoptotic role in cardiomyocyte apoptosis in part through the enhanced protein level of p27.

\section{Acknowledgements}

This work was supported by grants from the National Natural Science Foundation of China (81400204, 81401457, 81202187, 81300201, 8133003).

\section{Disclosure Statement}

None declared.

\section{Reference}

1 Kalanuria AA, Nyquist P, Ling G: The prevention and regression of atherosclerotic plaques: emerging treatments. Vasc Health Risk Manag 2012;8:549-561.

2 Dabek J, Owczarek A, Gasior Z, Ulczok R, Skowerski M, Kulach A, Mazurek U, Bochenek A: Oligonucleotidemicroarray analysis of genes regulating apoptosis in chronically ischemic and postinfarction myocardium. Biochem Genet 2008;46:241-247. Ordovas JM, Smith CE: Epigenetics and cardiovascular disease. Nat Rev Cardiol 2010;7:510-519. Lee JH, Gao C, Peng G, Greer C, Ren S, Wang Y, Xiao X: Analysis of transcriptome complexity through RNA sequencing in normal and failing murine hearts. Circ Res 2011;109:1332-1341.

-5 Calin GA, Liu CG, Ferracin M, Hyslop T, Spizzo R, Sevignani C, Fabbri M, Cimmino A, Lee EJ, Wojcik SE, Shimizu M, Tili E, Rossi S, Taccioli C, Pichiorri F, Liu X, Zupo S, Herlea V, Gramantieri L, Lanza G, Alder H, Rassenti L, Volinia S, Schmittgen TD, Kipps TJ, Negrini M, Croce CM: Ultraconserved regions encoding ncRNAs are altered in human leukemias and carcinomas. Cancer Cell 2007;12:215-229. Rinn JL, Chang HY: Genome regulation by long noncoding RNAs. Annu Rev Biochem 2012;81:145-166. Konstantinidis K, Whelan RS, Kitsis RN. Mechanisms of cell death in heart disease. Arterioscler Thromb Vasc Biol 2012;32:1552-1562.

8 Smith CC, Davidson SM, Lim SY, Simpkin JC, Hothersall JS, Yellon DM. Necrostatin: A potentially novel cardioprotective agent? Cardiovasc Drugs Ther 2007;21:227-233.

9 Accili D, Arden KC: Foxos at the crossroads of cellular metabolism, differentiation, and transformation. Cell 2004;117:421-426.

10 Huang J, Zhou N, Watabe K, Lu Z, Wu F, Xu M, Mo YY: Long non-coding RNA UCA1 promotes breast tumor growth by suppression of p27 (Kip1). Cell Death Dis. 2014;5:e1008.

$\checkmark 11$ Hengst L, Reed SI: Translational control of p27Kip1 accumulation during the cell cycle. Science 1996;271:1861-1864.

12 Wander SA, Zhao D, Slingerland JM: p27: a barometer of signaling deregulation and potential predictor of response to targeted therapies. Clin Cancer Res 2011;17:12-18.

13 Wang XS, Zhang Z, Wang HC, Cai JL, Xu QW, Li MQ: Rapid identification of UCA1 as a very sensitive and specific unique marker for human bladder carcinoma. Clin Cancer Res 2006;12:4851-4858.

14 Tsang WP, Wong TW, Cheung AH, Co CN, Kwok TT: Induction of drug resistance and transformation in human cancer cells by the noncoding RNA CUDR. RNA 2007;13:890-898.

15 Liu Y, Li G, Lu H, Li W, Li X, Liu H, Li X, Li T, Yu B. Expression profiling and ontology analysis of long noncoding RNAs in post-ischemic heart and their implied roles in ischemia/reperfusion injury. Gene 2014;543:15-21.

16 Tan WQ, Wang K, Lv DY, Li PF: Foxo3a inhibits cardiomyocyte hypertrophy through transactivating catalase. J Biol Chem 2008;283:29730-29739 


\section{Cellular Physiology Cell Physiol Biochem 2015;35:1986-1998

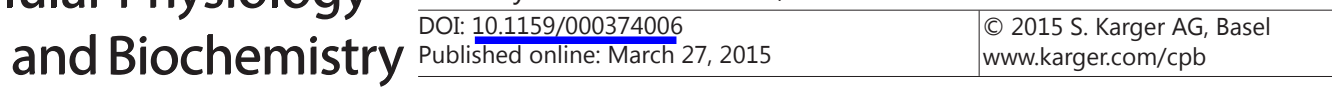

Liu et al.: Reduced UCA1 Level Leads to Cardiomyocyte Apoptosis

17 Braconi C, Valeri N, Kogure T, Gasparini P, Huang N, Nuovo GJ, Terracciano L, Croce CM, Patel T. Expression and functional role of a transcribed noncoding RNA with an ultraconserved element in hepatocellular carcinoma. Proc Natl Acad Sci U S A 2011;108:786-791.

18 Guo J, Li M, Meng X, Sui J, Dou L, Tang W, Huang X, Man Y, Wang S, Li J. MiR-291b-3p induces apoptosis in liver cell line NCTC1469 by reducing the level of RNA-bingding protein HuR. Cell Physiol Biochemistry. 2014;33:810-822.

19 Zhang S, Liu X, Bawa-Khalfe T, Lu LS, Lyu YL, Liu LF, Yeh ET: Identification of the molecular basis of doxorubicin-induced cardiotoxicity. Nat Med 2012;18:1639-1642.

20 Tuorkey MJ, EI-Desouki NI, Kamel RA: Cytoprotective effect of silymarin against diabetes-induced cardiomyocyte apoptosis in diabetic rats. Biomed Environ Sci 2015;28:36-43.

-21 Di Gesualdo F, Capaccioli S, Lulli M: A pathophysiological view of the long non-coding RNA world. Oncotarget 2014;5:10976-10996.

-22 Gazzaniga FS, Blackburn EH. An antiapoptotic role for telomerase RNA in human immune cells independent of telomere integrity or telomerase enzymatic activity. Blood 2014;124:3675-3684.

23 Lee B, Mazar J, Aftab MN, Qi F, Shelley J, Li JL, Govindarajan S, Valerio F, Rivera I, Thurn T, Tran TA, Kameh D, Patel V, Perera RJ: Long noncoding RNAs as putative biomarkers for prostate cancer detection. J Mol Diagn 2014;16:615-626.

24 Barnett P, van den Hoff MJ. Cardiac regeneration: different cells same goal. Med Biol Eng Comput 2011;49:723-32.

25 Koike M, Shibata M, Tadakoshi M, Gotoh K, Komatsu M, Waguri S: Inhibition of autophagy prevents hippocampal pyramidal neuron death after hypoxic-ischemic injury. Am J Pathol 2008;172:454-469.

-26 Puyal J, Vaslin A, Mottier V, Clarke PG: Postischemic treatment of neonatal cerebral ischemia should target autophagy. Ann Neurol 2009;66:378-389.

-27 Sun Y, Wang Y, Yin Y, Chen X, Sun Z : GSTM3 reverses the resistance of hepatoma cells to radiation by regulating the expression of cell cycle/apoptosis-related molecules. Oncol Lett 2014;8:1435-1440.

28 Wu S, Bao Y, Ma D, Zi Y, Yang C, Yang M, Xing M, Yang W: Sodium selenite inhibits leukemia HL-60 cell proliferation and induces cell apoptosis by enhancing the phosphorylation of JNK1 and increasing the expression of p21 and p27. Int J Mol Med 2014;34:1175-1179.

29 Slingerland J and Pagano M: Regulation of the cdk inhibitor p27 and its deregulation in cancer. J Cell Physiol 2000;183:10-17.

-30 Jatzek A, Tejera MM, Singh A, Sullivan JA, Plisch EH, Suresh M: p27(Kip1) negatively regulates the magnitude and persistence of CD4 T cell memory. J Immunol 2012;189:5119-5128. 